\title{
MICROMORFOLOGÍA Y ANATOMÍA DE FRUTOS Y SEMILLAS DE SCROPHULARIA L. EN EL SUROESTE DE ESPAÑA
}

\author{
Rocío JUAN, Julio PASTOR e Inmaculada FERNÁNDEZ
}

\begin{abstract}
RESUMEN. Micromorfología y anatomía de frutos y semillas de Scrophularia L. en el suroeste de España. Se ha realizado un estudio morfológico y anatómico de las cápsulas y semillas de las nueve especies del género Scrophularia presentes en el SO de España. Las semillas muestran una considerable uniformidad en cuanto a la forma, tamaño, ornamentación y estructura interna. Respecto a las cápsulas muestran ciertas diferencias tanto desde el punto de vista morfológico como anatómico. Por último se propone una clave para diferenciar las distintas especies.
\end{abstract}

Palabras clave. Fruto, Semilla, Anatomía, Morfología, Scrophularia, Scrophulariaceae

ABSTRACT. Micromorphology and anatomy of fruits and seeds of Scrophularia from south-west Spain. A morphological and anatomical study of fruits and seeds of nine species of Scrophularia from SW Spain has been made. The seeds show considerable uniformity in shape, size, seed-coat, ornamentation and internal structure. Respect to fruits show several differences from a morphological and anatomical view point. Finally a key to distinguish the different species is provided.

Key words. Fruit, Seed, Anatomy, Morphology, Scrophularia, Scrophulariaceae

\section{INTRODUCCIÓN}

El género Scrophularia lo constituyen alrededor de 300 especies de amplia distribución en el Hemisferio Norte (Carlbom, 1969). Según Stiefelhagen (1910, sec. Carlbom, 1969), el centro de diversificación y especiación fue la región del Himalaya. Aunque en una revisión reciente sobre este género en la Península Ibérica y Baleares, Ortega \& Devesa (1993) indican también la posibilidad de dos núcleos secundarios según el número de taxones: uno situado en la región de los Balcanes con 15 especies y otro en la Península
Ibérica con 29 especies (pertenecientes a las sects. Scrophularia y Canina). En Andalucía occidental se encuentran nueve especies, estando representadas las dos secciones (Valdés, 1987).

Son hierbas perennes, rara vez bienales o anuales, con hojas opuestas, de enteras a bipinnatisectas. Flores en cimas formando panículas o racimos más o menos terminales. Cáliz con 5 lóbulos iguales. Corola totalmente abierta con tubo subgloboso. Androceo con 4 estambres fértiles y 1 estaminodio rara vez ausente. Cápsulas septicidas. Semillas ovoides, con varias filas de alveolos transversales. 
La gran diversidad de adaptaciones fisiológicas a las distintas condiciones climáticas y edáficas en el género Scrophularia es un reflejo de la antigüedad de éste (Carlbom, 1969). El interés que tiene dicho género desde un punto de vista evolutivo, hace que sean frecuentes los estudios de carácter taxonómico, citotaxonómico o palinológicos como los de Carlbom (1969), Valsecchi (1977), Qaiser et al. (1988) y Ortega \& Devesa (1990, 1992, 1993 a) entre otros. Los frutos y semillas no han recibido otro tratamiento que el habitual dentro de los estudios taxonómicos, aunque merece una atención especial la contribución que hacen Bhandari et al. (1976) y Bhandari \& Natesh (1985), al conocimiento del desarrollo de la testa y el endospermo respectivamente en S. himalensis.

\section{MATERIAL Y MÉTODO}

El material estudiado procede en su mayoría de poblaciones recolectadas durante los años 1992 y 1993, aunque en algunas ocasiones se utilizó material prensado del Herbario del Departamento de Biología Vegetal y Ecología de la Universidad de Sevilla (SEV). El origen de las muestras examinadas se indica en el Apéndice.

Este material se fijó en FAA durante un mínimo de 24 horas pasándolo posteriormente a etanol al $70 \%$.

Para el estudio morfológico, algunas de las cápsulas y semillas se deshidrataron en una serie creciente de acetona y se sometieron a punto crítico usando dióxido de carbono. Dicho material junto a algunas semillas secas se montaron en portas mediante adhesivo de doble cara. A continuación se metalizaron con oropaladio y se examinaron con un microscopio electrónico de barrido (MEB) Philips LX-20. Con el fin de determinar si existía diferencia intra o interpoblacional se aclararon y rebladencieron, en ácido láctico, varias cápsulas de cada población para comparar las epidermis. Éstas se montaron sobre portas y se observaron al microscopio óptico (MO). Los datos de longitud y anchura están basados en el muestreo de 35 a 120 cápsulas o semillas por taxón. Con ayuda de una cámara clara se realizaron dibujos de las cápsulas completas.

Del mismo modo, para el estudio anatómico se ha usado material fijado, que posteriormente se deshidrató usando las series de alcohol butílico terciario (Johansen, 1940). A continuación se incluyó en parafina y se cortó a 8 - $10 \mu \mathrm{m}$. Una vez montadas las secciones, se tiñeron con safranina alcohólica al $1 \%$ y fast green alcohólico al 0,1\%. Estas preparaciones se montaron de modo permanente para su posterior observación al MO.

Para la terminología se ha seguido fundamentalmente a Font Quer (1993) y a Stearn (1992) .

Los testigos se encuentran depositados en el Herbario del Departamento de Biología Vegetal y Ecología de la Facultad de Biología de Sevilla (SEV).

\section{Material estudiado.}

S. canina L. subsp. canina. CÁDIZ. Bornos, 7.5.1992, Arista \& Talavera (SEV 135249). CORDOBA. Entre Carcabuey y Luque, 23.5.1992, Arista, Juan, Ortiz \& Santa-Bárbara (SEV 135250). Los Blázquez, 9.6.1992, Juan \& Pastor (SEV 135251). HUELVA. Entre la Palma del Condado y Valverde del Camino, 25.4.1992, Juan \& López (SEV 135252). Entre Almonaster la Real y el cruce de la carretera de Huelva, 18.5.1992, Juan (SEV 135253). Río Tinto, arroyo, 5.6.1992, Juan, Pastor \& Santa-Bárbara (SEV 135254).

S. crithmifolia Boiss. CÁDIZ. Grazalema, El Torreón, 30.4.1992, Arista (SEV 135255). Ídem, Puerto de las Palomas, 7.5.1992, Arista \& Talavera (SEV 135256). Ídem, Puerto del Boyar, 27.5 1992, Juan \& Pastor (SEV 135257).

S. frutescens L. CÁDIZ. Punta Palomas, 1.7.1993, Diosdado, Juan \& Pastor (SEV 135258). HUELVA. Entre Matalascañas y Mazagón, 7.5.1992, Juan \& López (SEV 135259). Entre 
Mazagón y Huelva, 7.5.1992, Juan \& López (SEV 135260). Matalascañas, 7.6.1992, Fernández \& Juan (SEV 135261).

S. laevigata Vahl. CÁDIZ. Los Barrios, El Tiradero, 6.6.1992, Ojeda (SEV 135233). Facinas, 11.6.1992, Fernández \& Juan (SEV 135234).

S. lyrata Willd. CÓRDOBA. Entre Torrecampo y Guijo, 8.6.1978, Domínguez, Muñoz \& Valera (SEV 98357). Entre los Blázquez y Peraleda del Zaucejo, 6.6.1979, Muñoz \& Ruizde Clavijo (SEV 103986). HUELVA. Entre Zufre y la Junta Ribera de Huelva, 15.5.1979, Rivera (SEV 48623). Fuenteheridos, 18.5.1992, Juan (SEV 135231). Peña de Arias Montano, 18.5.1992, Juan (SEV 135232)

S. oxyrhyncha Coincy. CÓRDOBA. Entre Santa Eufemia y la estación de Belalcázar, 6.5.1992, Juan (SEV 135235).

S. peregrina L. GRANADA. Entre Almuñécar y Jete, 4.6.1992, Juan \& Pastor (SEV 135236). MÁlAGA. Yunquera, 18.4.1973, Talavera \& Valdés (SEV 102093). Valle de Abdalajís, 18.6.1973, Talavera \& Valdés (SEV 102094).

S. sambucifolia L. subsp. mellifera (H'Hér. ex Aiton) Maire. CÁDIZ. La Almoraima, 11.6.1992, Fernández \& Juan (SEV 135247). Entre Medina Sidonia y Los Barrios, 15.6.1993, Fernández \& Juan (SEV 135248).

S. sambucifolia L. subsp. sambucifolia. CÁDIZ. Entre Tarifa y Algeciras, 27.4.1992, Arista \& Juan (SEV 135242). Entre Vejer de la Frontera y Tarifa, 27.4.1992, Arista \& Juan (SEV 135243). Zahara de la Sierra, 20.4.1992, Arista \& Juan (SEV 135244). Villaluenga del Rosario, 27.5.1992, Juan \& Pastor (SEV 135245). HUELVA. Entre Beas y Candón, 7.6.1993, Fernández \& Juan (SEV 135246).

S. scorodonia L. CÁDIZ. Grazalema, La Camilla, 20.4.1992, Arista \& Juan (SEV 135237). Ídem, Llano del Revés, 17.6.1992, Arista \& Juan (SEV 135238). HUElva. Castaño del Robledo, 18.5.1992, Juan (SEV 135239). Entre Almonaster la Real y el cruce con la carretera de Huelva, 15.8.1992, Juan \& López (SEV 135240). MÁLAGA. Estepona, Puerto de Peñas Blancas, 21.5.1992, Arista \& Juan (SEV 135241).

\section{RESULTADOS}

Cápsulas glabras, de tamaño variable (3.5 - 13.6 x 3.0 - $10.0 \mathrm{~mm}$ ), leñosas, dispuestas en cimas. Ovoideas, subglobosas o subcónicas, con ápice apiculado salvo en $S$. oxyrhyncha que es acuminado; simetría radiada. Lóculos subiguales, paralelos y septo recto (fig. 1 a-i). Estilo no persistente. Superficie con células poligonales y cutícula lisa, estriada o estriadarugulada; con estomas (fig. 2 a-c). Pericarpo de grosor variable $(120-540 \mu \mathrm{m})$. Epicarpo formado por células pequeñas de paredes delgadas. Mesocarpo con 1 ó 2 regiones diferenciables. Cuando presenta dos zonas, la externa está formada por una capa de células de mayor tamaño y la interna por varias capas de células más pequeñas y frecuentemente colapsadas. Endocarpo de grosor variable (50 $-350 \mu \mathrm{m})$, donde se pueden diferenciar 2 ó 3 regiones atendiendo a la orientación de las células (fig. d,e). Dehiscencia septicida, sobrepasando la mitad del fruto. El número de semillas por cápsulas varía entre (6-) 8 - 450 (tab. 1).

Semillas de tamaño variable $(0.3-1.6 \mathrm{x}$ 0.3 - $1.1 \mathrm{~mm}$ ), ovoideas, oblongoideas o elipsoideas, a veces prismáticas o deprimidas. Inserción apical. Color de pardo obscuro a negro. Testa con alvéolos dispuestos en $6-8$ filas longitudinales. Superficie cubierta por una capa de células alargadas tangencialmente, con paredes radiales poco profundas y grosor irregular, dando un aspecto de malla; pared tangencial externa membranosa, colapsada sobre la interna que presenta engrosamientos escalariformes. Epidermis con células oblongas de paredes reforzadas excepto la tangencial externa. Endotelio reducido a una capa de cutina (fig. 2 f-i). No se ha observado rafe (tab. 1).

\section{Clave de frutos}

1. Cápsula $>9.0 \mathrm{~mm}$ de longitud. Pericarpo $>350$ $\mu \mathrm{m}$....................................... S. sambucifolia s.l.

1'. Cápsula $<9.0 \mathrm{~mm}$ de longitud. Pericarpo generalmente $<350 \mu \mathrm{m}$ 2

2. Cápsula subcónica S. oxyrhyncha

2'. Cápsula ovoidea o subglobosa ............................. 3

3. Mesocarpo diferenciado en 2 regiones.............. 4 

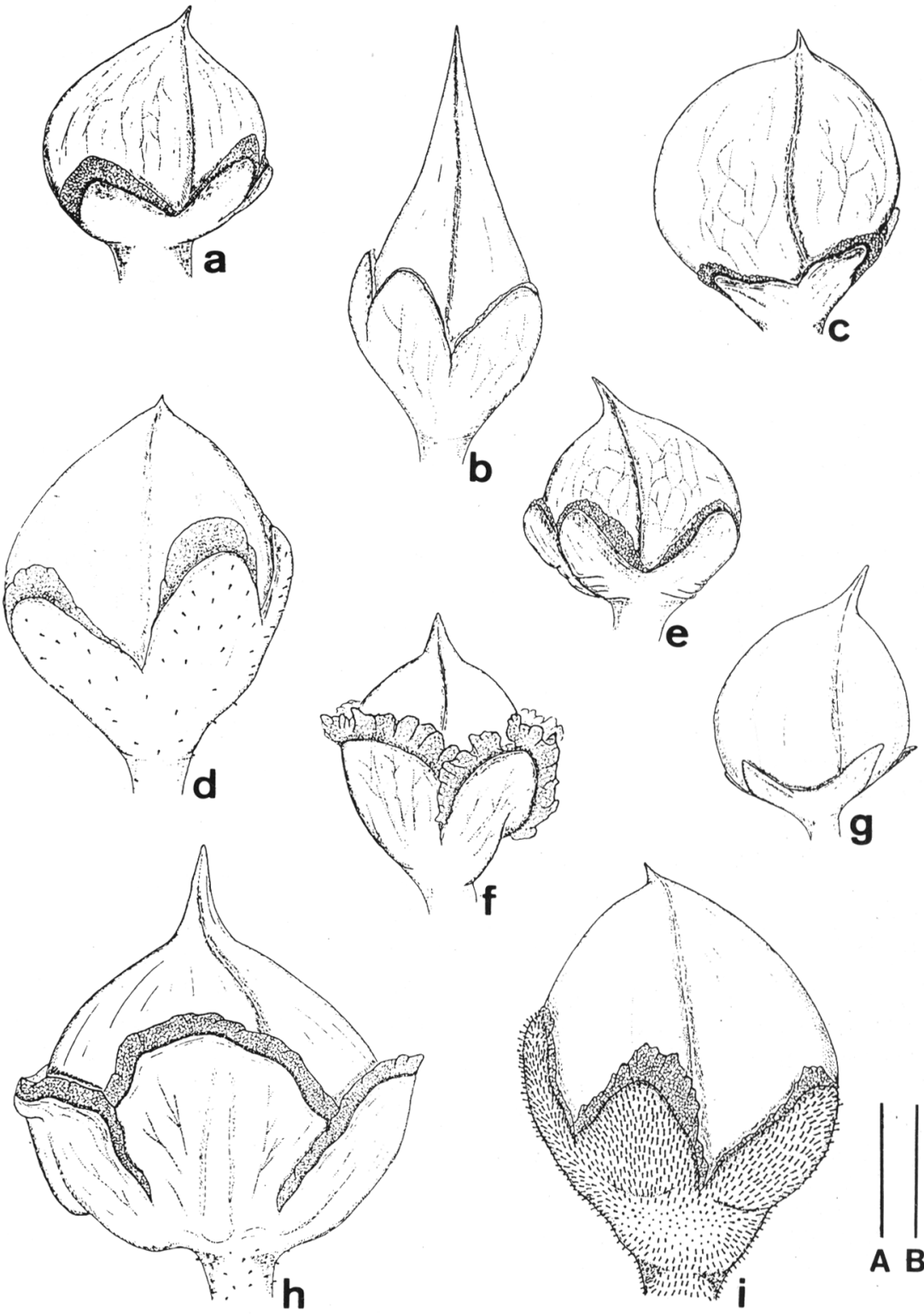

P.J. López

Figura 1. Cápsulas de Scrophularia: a; S. canina subsp. canina: b; S. oxyrhyncha: c; S. frutescens : d; S. laevigata: e; S. lyrata: f; S. crithmifolia: g; S. peregrina: h; S. sambucifolia subsp. sambucifolia: i; $S$. scorodonia. Escalas: $\mathrm{A}=4 \mathrm{~mm}(\mathrm{~h}) ; \mathrm{B}=3 \mathrm{~mm}(\mathrm{a}-\mathrm{g}, \mathrm{i})$. Drawing of capsules of Scrophularia: $a$; $S$. canina subsp. canina: $b$; S. oxyrhyncha: c; S. frutescens : d; S. laevigata: $e$; S. lyrata: $f$; $S$. crithmifolia: $g$; $S$. peregrina: $h$; S. sambucifolia subsp. sambucifolia: $i$; S. scorodonia. Scale bars: $A=4 \mathrm{~mm}(h) ; B=3 \mathrm{~mm}$ $(a-g, i)$. 

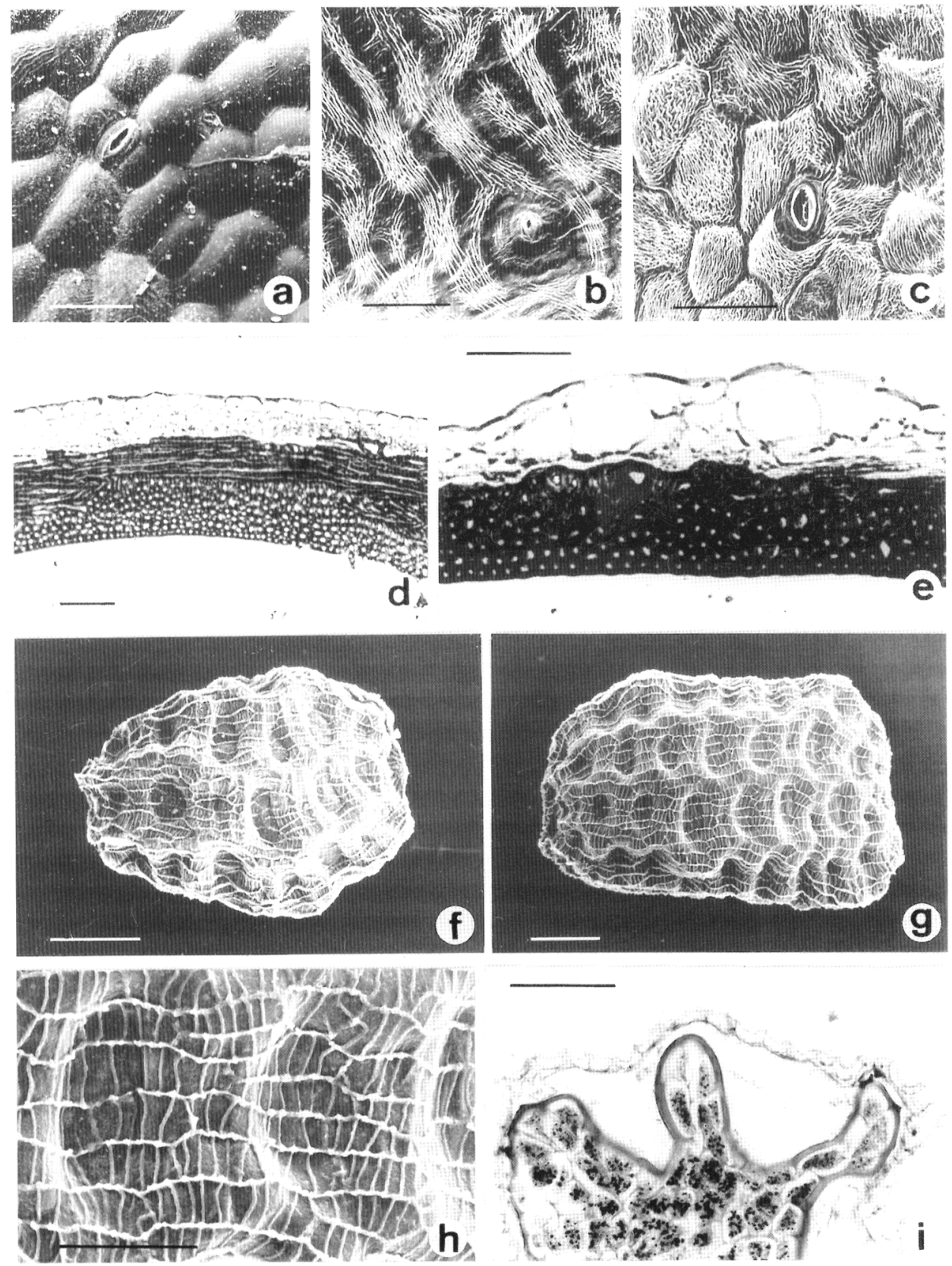

Figura 2. S. sambucifolia subsp. sambucifolia: a; S. lyrata: b, e, f; S. oxyrhyncha: c; S. frutescens: d; S. laevigata: g, h; S. scorodonia: i. a, b, c: superficie de la cápsula; d, e: detalle del pericarpo; f, g: aspecto general de la semilla; h: superficie de la testa; i: detalle de la testa. Escalas: $50 \mu \mathrm{m}$ (b, c, e); $100 \mu \mathrm{m}$ (a, d, $\mathrm{f}, \mathrm{h}, \mathrm{i}) ; 200 \mu \mathrm{m}(\mathrm{g})$. S. sambucifolia subsp. sambucifolia: $a$; S. lyrata: $b, e, f ;$ : oxyrhyncha: $c$; S. frutescens: $d$; S. laevigata: $g, h ; S$. scorodonia: $i . a, b, c$ : surface of capsule; $d, e$ : detail of pericarp; $f, g$ : whole seed; h: surface of seed-coat; $i$ : detail of seed-coat. Scale bars: $50 \mu \mathrm{m}(b, c, e) ; 100 \mu \mathrm{m}(a, d, f, h, i) ; 200 \mu \mathrm{m}(\mathrm{g})$. 
3'. Mesocarpo no diferenciado en 2 regiones ...... 5

4. Endocarpo $<120 \mu \mathrm{m}$, con 7 o menos capas de células ................................................... S. lyrata

4'. Endocarpo $>120 \mu \mathrm{m}$, con 7 o más capas de células ..................... S. laevigata, S. scorodonia

5. Cápsula con células convexas y cutícula estriada. Con más de 60 semillas. $S$. peregrina

5'. Cápsula con células planas y cutícula lisa o estriado-rugulosa. Con menos de 60 semillas. 6

6. Pericarpo $<200 \mu \mathrm{m}$ S. crithmifolia

6'. Pericarpo $>200 \mu \mathrm{m}$ .. S. canina subsp. canina, S. frutescens

\section{DISCUSIÓN}

Resulta evidente la separación de dos secciones en este género basadas en diversos caracteres morfológicos, cariológicos, ecológicos, etc. (Carlbom, 1969). Sin embargo, al considerar de forma aislada los caracteres derivados de frutos y semillas es difícil diferenciar ambos grupos, si bien las especies estudiadas de la sect. Canina se caracterizan por presentar un número menor de semillas por cápsula $(<50)$.

A pesar de que Valdecchi (1977) considera los frutos, entre otros caracteres, como constantes y bien diferenciados para cada especie, después del estudio realizado, se ha comprobado que morfológicamente sólo se pueden diferenciar tres grupos entre los taxones examinados. El primero con cápsulas subcónicas incluye únicamente $S$. oxyrhyncha, el segundo las tiene claramente ovoideas $(S$. laevigata y $S$. scorodonia) y el tercer y último grupo, donde se incluyen los restantes taxones, es el más complejo ya que las cápsulas son mayoritariamente subglobosas pero a veces también pueden ser ovoideas. En este último grupo serían necesarios otros caracteres adicionales para una identificación morfológica. Por otro lado, los caracteres anatómicos han resultado de gran interés en este género, permitiendo caracterizar la mayoría de las especies. Así, el mesocarpo puede aparecer diferenciado en dos regiones atendiendo al tamaño de las células (S. lyrata, $S$. laevigata y $S$. scorodonia) o bien estar indiferenciado como ocurre en $S$. peregrina y en todas las especies de la sect. Canina.

El grosor del pericarpo y del endocarpo también ha sido de utilidad, aunque insuficientes para diferenciar $S$. laevigata de $S$. scorodonia, y $S$. canina s.s. de $S$. frutescens. En estas dos últimas especies, a pesar de tener cápsulas muy semejantes, se observa una tendencia en $S$. frutescens a presentar frutos más robustos. El endocarpo en este género es bastante complejo, ya que suele tener un número alto de capas de células y orientadas de diferentes formas. Dicha orientación puede variar dependiendo de la zona del fruto, así en la mitad superior las células se suelen disponer perpendiculares al corte longitudinal, mientras que en la mitad inferior lo hacen en sentido paralelo. Ésto, a medida que la cápsula se seca, crea tensiones haciendo que estas células se acorten. De este modo, las que se disponen de forma paralela provocan la separación de ambos lóculos (dehiscencia septicida) y las que están en sentido perpendicular facilitarán la contracción de los márgenes de cada lóculo, curvándose hacia dentro. Este fenómeno ha sido anteriormente observado en Verbascum (Juan et al., 1997).

Las semillas, por otra parte, son muy características aunque no exclusivas en la familia. No se han observado entre ellas diferencias interespecíficas, aunque sí algunas ligeras desigualdades en forma y tamaño que resultan insuficientes para una clara identificación. Así, atendiendo al tamaño, las especies de la sect. Canina tienen las semillas más grandes y $S$. lyrata (sect. Scrophularia) presenta las semillas más pequeñas, aunque al ser el rango de variabilidad muy alto las medidas se solapan. En un estudio realizado por Bhandari et al. (1976) sobre S. himalensis, observaron que la superficie de las semillas 


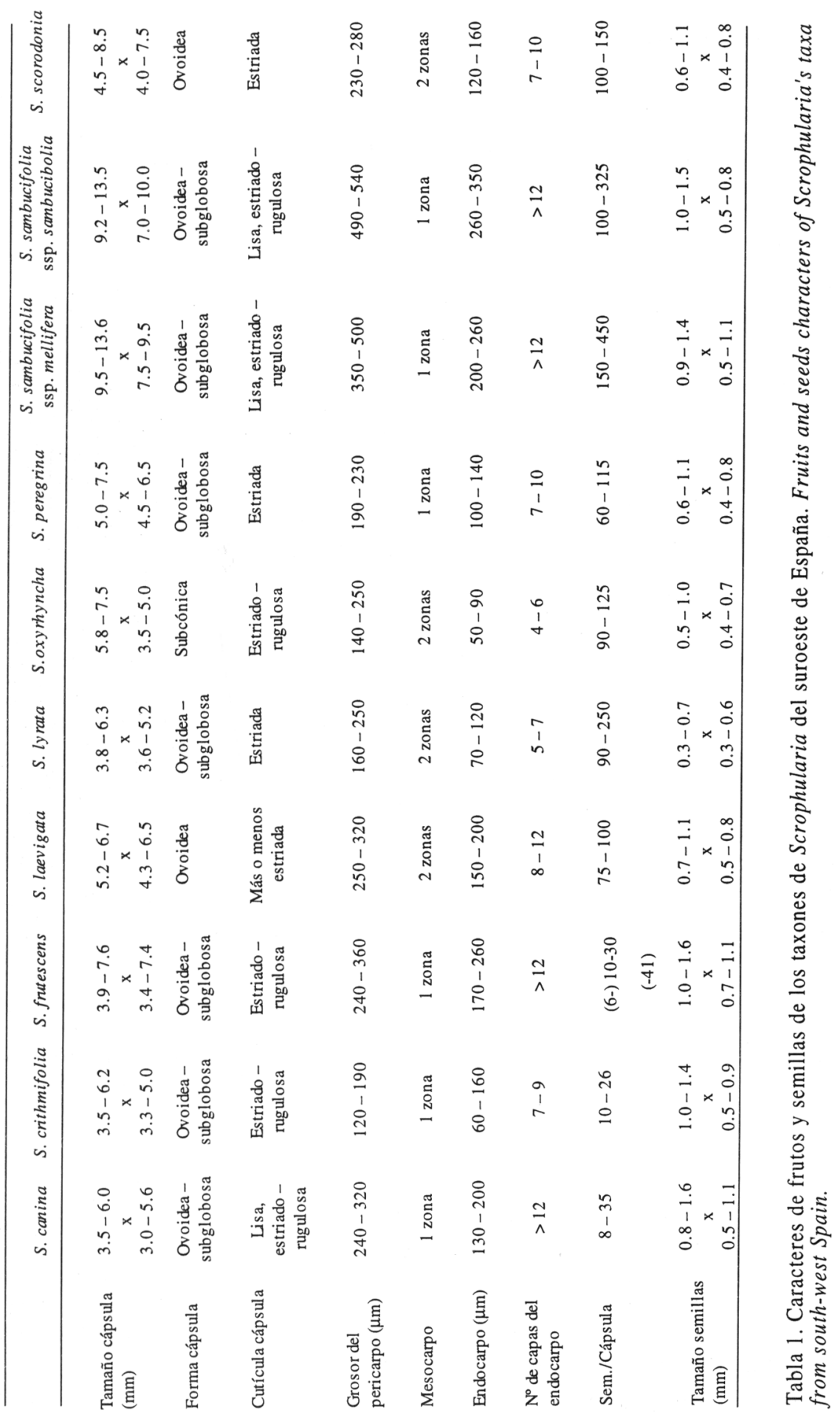


mostraban crestas y surcos muy característicos. En el presente estudio se ha considerado que las semillas de este género muestran de seis a ocho filas longitudinales de alvéolos, aunque cuando la semilla está hidratada no se aprecian. Según Bhandari et al. (1976) la apariencia externa en las semillas de $S$. himalensis es un fiel reflejo de su estructura interna, ya que el número de crestas es igual al número de células endoteliales observadas en corte transversal. De acuerdo con estos autores, la testa está constituida por la epidermis y el endotelio. La epidermis a su vez la forman células cuyas paredes radiales y pared tangencial interna presentan algunos engrosamientos de apariencia escalariforme, posiblemente de polisacáridos no solubles que parecen servir de soporte mecánico a las semillas. Durante la maduración de las semillas, las células del endotelio aumentan de tamaño pero no en número, originando un endospermo con apariencia lobulada o ruminada como ocurre en el género Verbascum (Periasamy, 1966; Juan et al., 1997). El contenido celular de las células endoteliales desaparece en poco tiempo quedando sólo una capa de cutina sobre la pared tangencial interna. Bhandari et al. (1976) indican que el endotelio, en $S$. himalensis, sirve de alimento al saco embrionario durante los estadios más tempranos pero después adquieren una función protectora. Ésto parece evidente, en los taxones estudiados en el presente trabajo, teniendo en cuenta la pérdida del contenida celular y la deposición de una capa de cutina.

En el género Scrophularia, la dispersión pasiva parece la más adecuada. La disposición de las cápsulas y la dehiscencia de éstas, entre otras cosas, harán que las semillas permanezcan en las mismas hasta que algún agente externo provoque el movimiento de las ramas. Pijl (1982), considera que el viento es el principal vector por lo que estas plantas serían consideradas anemobalistas. No obstante, Ortega \& Devesa (1993 b), indican que las hormigas también intervienen en la dispersión, ya que transportan cápsulas completas después de haberlas cortado. Por otro lado, las semillas presentan gran capacidad de hidratación que podría ser aprovechada en la germinación, como ya ha puesto de manifiesto Sutton (1988) en otros géneros de Scrophulariaceae.

AGRADECIMIENTOS. Los autores agradecen al Servicio de Microscopía Electrónica de la Universidad de Sevilla la ayuda prestada, especialmente a Dña. A. Fernández por la preparación de las muestras en punto crítico. Asimismo, queremos agradecer al Dr. P. J. LópezGonzález la realización de los dibujos que aparecen en la publicación.

\section{BIBLIOGRAFÍA}

BHANDARI, N. N. \& S. NATESH -1985Development of endosperm in Scrophularia himalensis with a discussion on the variation in the endosperm of the tribe Scrophularieae (Scrophulariaceae). Pl. Sys. Evol. 148: 177184.

BHANDARI, N. N., F. BOUMAN \& S. NATESH 1976- Ovule ontogeny and seed coat structurea of Scrophularia himalensis Royle. Bot. Jahrb. Syst. 95: 535-548.

CARLBOM, C. -1969- Evolutionary relationships in the genus Scrophularia L. Hereditas 61: 287 301.

FONT QUER, P. -1993-Diccionario de Botánica. Barcelona.

JUAN, R., I. FERNÁNDEZ \& J. PASTOR -1997Systematic consideration of fruits and seeds in the genus Verbascum (Scrophulariaceae). Ann. Bot. 80: 591-598.

ORTEGA, A. y J. A. DEVESA - 1990- Contribución al estudio cariológico del género Scrophularia L. (Scrophulariaceae) en la Península Ibérica y Baleares. Lagascalia 16: 171-198.

ORTEGA, A. y J. A. DEVESA -1992- Estudio palinológico del género Scrophularia en la Península Ibérica e Islas Baleares. Acta Bot. Malacitana 17: 195-207.

ORTEGA, A. y J. A. DEVESA - 1993 a-Revisión del 
género Scrophularia L. (Scrophulariaceae) en la Península Ibérica e Islas Baleares. Ruizia 11: 1-157.

ORTEGA, A. \& J. A. DEvESA -1993 b- Sexual reproduction in some Scrophularia species (Scrophulariaceae) from the Iberian Peninsula and the Balearic Islands. Pl. Syst. Evol. 184: 159-174.

PERIASAMY, K. -1962- The ruminate endosperm development and types of rumination, 62-74. In P. MAHESHWARI (ed.) Plant Embryology. India.

PIJL, L., VAN DER -1982- Principles of Dispersal in Higher Plants. Berlín.

QAISER, M., S. KHATOON \& HAMIDULLAH 1988- The genus Scrophularia (Scrophulariaceae) in Pakistan. Willdenowia 17: 129-146.

STEARN, W. T. -1992-Botanical Latin. London.
SUTTON, D. A. $-1988-$ A revision of the tribe Antirrhineae (Scrophulariaceae). Oxford.

VALDÉS, B. -1987- Scrophulariaceae. In B. VALDÉS et al. (eds.). Flora vascular de Andalucía Occidental. Vol. 2. Barcelona.

VALSECCHI, F. -1977- Observations sur quelques espèces du genre Scrophularia L. en Sardaigne. Webbia 34: 286-287.

Aceptado para su publicación en Septiembre de 1999

Dirección de los autores. Dpto. Biología Vegetal y Ecología. Facultad de Farmacia. Universidad de Sevilla. 41012, Sevilla. 